\title{
KİTOSAN/ter(GMA-MA-NTBA) POLİMER İLE MİKROKAPSÜL HAZIRLAMA VE KARAKTERIZASYONU
}

\author{
İdris SARGIN \\ Selçuk Üniversitesi, Fen Fakültesi, Biyokimya Bölümü, Kampüs-Konya, TÜRKIYYE \\ idris.sargin@selcuk.edu.tr
}

(Geliş/Received: 24.12.2018; Kabul/Accepted in Revised Form: 05.02.2019)

\begin{abstract}
ÖZ: Bu çalışmada, glisidil metakrilat (GMA), maleik anhidrit (MA), N-ter-butilakrilamid (NTBA) monomerlerinden ter(GMA-MA-NTBA) polimer ilk kez sentezlenmiştir. Polimerin kimyasal yapısı FTIR spektroskopisi, Nükleer Manyetik Rezonans ( $\left.{ }^{1} \mathrm{H}-\mathrm{NMR}\right)$ spektroskopisi, asit sayısı ve viskozite ölçümleri ile yüzey morfolojisi taramalı elektron mikroskobu (SEM) ile çalışılmıştır. Sentezlenen ter(GMA-MA-NTBA) polimerin farklı oranlarını içeren kitosan tabanlı mikrokapsüller hazırlanmış ve yapısal kararlılığı optimize edilmiştir. Polimer-kitosan mikrokapsüller gluteraldehit çapraz bağlayıcı ile daha dayanıklı hale getirilmiştir. Mikrokapsüllerin kimyasal yapıları FT-IR spektroskopisi ile yüzey morfolojileri SEM ile belirlenmiştir. Kitosan/ter(GMA-MA-NTBA) polimer kompozit mikrokapsüller farklı adsorpsiyon, ilaç taşıma ya da kontrollü salınım uygulamalarında denenebilir.
\end{abstract}

Anahtar Kelimeler: Kitosan mikrokapsül, ter-Polimer, SEM

\section{Preparation and Characterization of Chitosan/ter(GMA-MA-NTBA) Polymer Microcapsules}

ABSTRACT: In this study, ter(GMA-MA-NTBA) polymer was synthesized from glycidyl methacrylate(GA); maleic anhydride (MA) and N-tert-butylacrylamide (NTBA) monomers for the first time. Chemical structure of the polymer was studied using FT-IR spectroscopy, Nuclear Magnetic Resonance ( $\left.{ }^{1} \mathrm{H}-\mathrm{NMR}\right)$ spectroscopy, acidic group number and viscosity measurements and its surface morphology using scanning electron microscopy (SEM). Chitosan-based microcapsules containing different ratios of the synthesized ter(GMA-MA-NTBA) polymer were prepared and their structural stability was optimized. The microcapsules were cross-linked with glutaraldehyde to make them mechanically stronger. Chemical structure of the microcapsules was determined using FT-IR spectroscopy and their surface their surface morphology using SEM. Chitosan/ter(GMA-MA-NTBA) polymer composite microcapsules can be tested in adsorption, drug delivery and controlled release applications.

Key Words: Chitosan microcapsule, ter-Polymer, SEM

\section{GİRIŞ (INTRODUCTION)}

Polimerik malzeme içeren kitosan tabanlı mikrokapsül hazırlamada, genelde üç metot kullanılmıştır. İlki; polimerin doğrudan kitosanla beraber harmanlanıp karıştırılmasıyla oluşan hidrojel çözeltinin jelleştirmesiyle elde edilen mikrokapsüllerdir. Mikrokapsül üretimi kitosanın alkali ortamda tekrar jelleştirilmesine dayanır ve kitosan-polimer ya da kitosan-kitosan arasında kovalent bir bağlanma gerçekleşmez, iki polimerden oluşan bir karışım elde edilir (Won ve diğ., 2009; Tran ve diğ., 2013). İkincisi; sentetik monomerin kitosan çözeltisi içerisinde polimerleştirilmesiyle elde edilir. Bu tip mikrokapsül üretiminde polimer monomerinden, kitosan çözeltisi içerisinde in situ olarak sentezlenir ve oluşan hidrojel karışım semi-IPN (Semi-interpenetrating polymer network) olarak isimlendirilir. Polimer alaşımlar olarak nitelendirilebilecek bu polimerlerle; kontrollü salınım için mikroküreler (Rokhade ve 
diğ., 2007), sulu çözeltilerden metal (Akkaya ve Ulusoy, 2008; Wang ve diğ., 2013) ve boya (Zhao ve diğ., 2012) sorpsiyonu için adsorbanlar, biyouyumlu jel matriksler (Zhou ve $\mathrm{Wu}, 2011$ ) ve pH duyarlı semiIPN mikroküreler (Babu ve diğ., 2008) hazırlanmıştır. Son olarak, sentetik monomerin polimerleşmesini takiben kitosan zincirlerinin çapraz bağlayıcıyla bağlanmasıyla elde edilen full-IPN'lerdir (Fullinterpenetrating polymer network). Bu polimerlerle; $\mathrm{pH}$ ve sıcaklık duyarlı aşı polimerler (Don ve Chen, 2005), sensörler (Kestwal ve diğ., 2011), boyar maddelerin uzaklaştırılması için sorbentler (Dragan ve

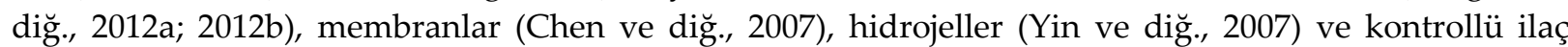
salınımı için jel matriksler (Agnihotri ve Aminabhavi, 2006) üretilmiştir. İfade edilen kullanım alanlarında ve çalışmalarda, kitosan; membran (Bayramoglu ve diğ., 2007), film (Sharmin ve diğg., 2012; Dotto ve diğg., 2013), fiber (Pillai ve diğ., 2009), nanopartikül (Liu ve diğ., 2012; Wei ve diğg., 2012) hidrojel (Pal ve diğ., 2013), reçine (Atia, 2005; Abou El-Reash ve diğ., 2011), nano/mikroküreler (Chen ve diğ., 2008) veya kapsüller (Kumar ve diğ., 2004; Ma ve diğ., 2009) halinde kullanılmıştır.

Kitosan tek başına zayıf bir bazdır ( $\mathrm{pKa} \sim 6.2$ ) ve $\mathrm{pH}<6.0^{\prime}$ nın altında asidik ortamlarda çözünür. Fakat kitosanın çapraz bağlamayla modifiye edilmesi bu sınırlılığı ortadan kaldırır (Guibal ve diğg., 1995; Piron ve diğ., 1997; Anirudhan ve Rijith, 2009). Literatürde ham kitosanın organik türe karşı seçici hale getirmek için de çeşitli modifikasyon yöntemleriyle farklı kitosan türevleri sentezlenmiştir (Xu ve diğ., 2013). Kitosanın deasetilasyon derecesi, molekül ağırlığı ve kristal yapısı gibi fizikokimyasal özellikleri, izole edildiği kaynağa ve izolasyon yöntemine son derece bağlıdır. Bunun yanında kitosanın sentetik polimerle beraber kullanılmasında sentetik polimerin de kimyasal ve fiziksel yapısının hesaba katılması bir zorunluluktur. Bütün parametrelerinin aynı reaksiyon ortamında optimize edilmesi zordur.

$\mathrm{Bu}$ çalışmada, farklı organik moleküller için taşıyıcı ya da adsorban olarak kullanılabilecek kitosan tabanlı bir kompozit mikrokapsül üretimi amaçlanmıştır. Çalışmada ter(GMA-MA-NTBA) polimeri hazırlanıp kitosanla beraber mikrokapsül hazırlamada kullanılmıştır. Hazırlanan ter-polimerle kitosanın farklı oranlarda karıştırıldıktan sonra kitosanın çapraz bağlanmasıyla kompozit kitosan/ter(GMA-MANTBA) polimer mikrokapsüller ilk kez üretildi. Kitosan çözeltisine katılan ter(GMA-MA-NTBA) polimeri sentezlenip kitosan çözeltisine katılmıştır. Bu anlamda yukarıda ifade edilen mikrokapsül hazırlama yöntemlerinden birinci yönteme benzemektedir. Diğer yandan ter(GMA-MA-NTBA) polimerin taneciklerinin kitosan çözeltisine ilave edilmesinden kitosanın çapraz bağlanmasıyla semiIPN'lere kısmen bir benzerlik söz konusudur. Sentezlenen ter(GMA-MA-NTBA) polimerin kimyasal yapısı FT-IR spektroskopisi, Nükleer Manyetik Rezonans ('H-NMR) spektroskopisi ile analiz edilmiş ve polimerin asit sayısı, viskozitesi ve taramalı elektron mikroskobu (SEM) ile de yüzey morfolojisi incelenmiştir. Kitosan/ter(GMA-MA-NTBA) polimer mikrokapsüllerin kimyasal yapısı FT-IR spektroskopisi ile yüzey morfolojileri SEM ile incelenmiştir.

\section{ter-POLIMERIN SENTEZLENMESİ VE KARAKTERIZASYONU (SYNTHESIS AND CHARACTERIZATION OF TER-POLYMER)}

ter(GMA-MA-NTBA) (1:2:1), N-ter-butilakrilamid (NTBA), maleik anhidrit (MA) ve glisidil metakrilat (GMA) monomerlerinden inert atmosfer ortamında radikalik polimerizasyon yöntemiyle sentezlenmiştir (Şekil 1) (Nie ve diğ., 2004; Wu ve diğ., 2006; Klumperman, 2010; Rzaev, 1999; Kaplan ve Rzaev, 2012; Dinçer ve diğ., 2002). 0.01 mol NTBA (1.27 g), 0.02 mol MA (1.96 g) ve 0.01 mol GMA (1.42 g) 1,4-dioksan $(40 \mathrm{~mL})$ içine ilave edildikten sonra üç boyunlu balon içerisinde 30 dakika azot gazı geçirildi. Benzoil peroksit (BPO) $\left(4 \times 10^{-4} \mathrm{~mol}\right)$ ilavesinden sonra karışım $85^{\circ} \mathrm{C}^{\prime}$ de 20 saat süreyle ısıtıldı (Şekil 1). Sonrasında, karışım çözücünün fazlasını ortamdan uzaklaştırmak için evaporatöre alındı. Ortamda kalan reaktif ve monomerleri uzaklaştırmak için karşım dietil eter içerisine damla damla ilave edildi, çöken polimer ortamdan alınıp tekrar 1,4-dioksan içerisinde çözüldü ve çözelti tekrar dietil eter içerisinde tekrar çöktürüldü. Üçüncü tekrardan sonra saflaştırılan polimer çökeltisi ortamdan alınıp, çözücü uzaklaştırıldıktan sonra kullanıldı.

Sentezlenen ter(GMA-MA-NTBA) polimer; Infread Spektroskopi (FT-IR) (Bruker Vertex 70 FTIR spektrometre; 4000-500 $\mathrm{cm}^{-1}$.), Nükleer Manyetik Rezonans ('H-NMR) (Varian $400 \mathrm{MR}$ ), Taramalı 
Elektron Mikroskobu (SEM) (EVO LS 10 ZEISS), asit sayısı titrimetrik yöntemle, Viskozimetre (Ubbelohde Viskozimetresi) ile karakterize edilmiştir.
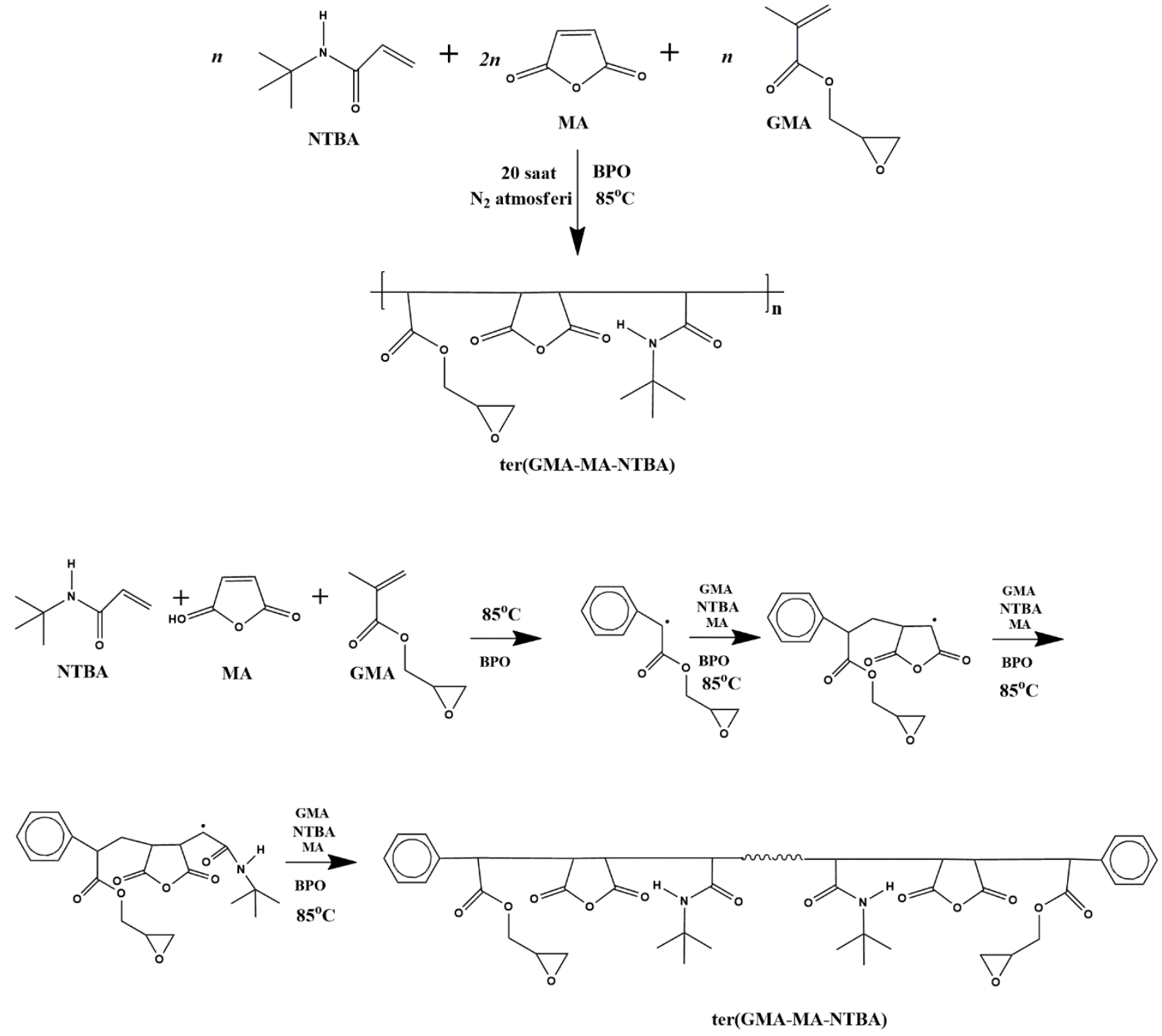

Şekil 1. ter(GMA-MA-NTBA) polimerinin muhtemel oluşum mekanizması Figure 1. A possible mechanism for synthesis of tert-(GMA-MA-NTBA) polymer

\section{ter(GMA-MA-NTBA)'1n FT-IR spektrumu (FT-IR spectrum of ter(GMA-MA-NTBA))}

Sentezlenen ter(GMA-MA-NTBA) polimeri; Infrared Spektroskopi (FT-IR) ile analiz edilmiş ve FTIR spektrumu Şekil 2' de verilmiştir. 


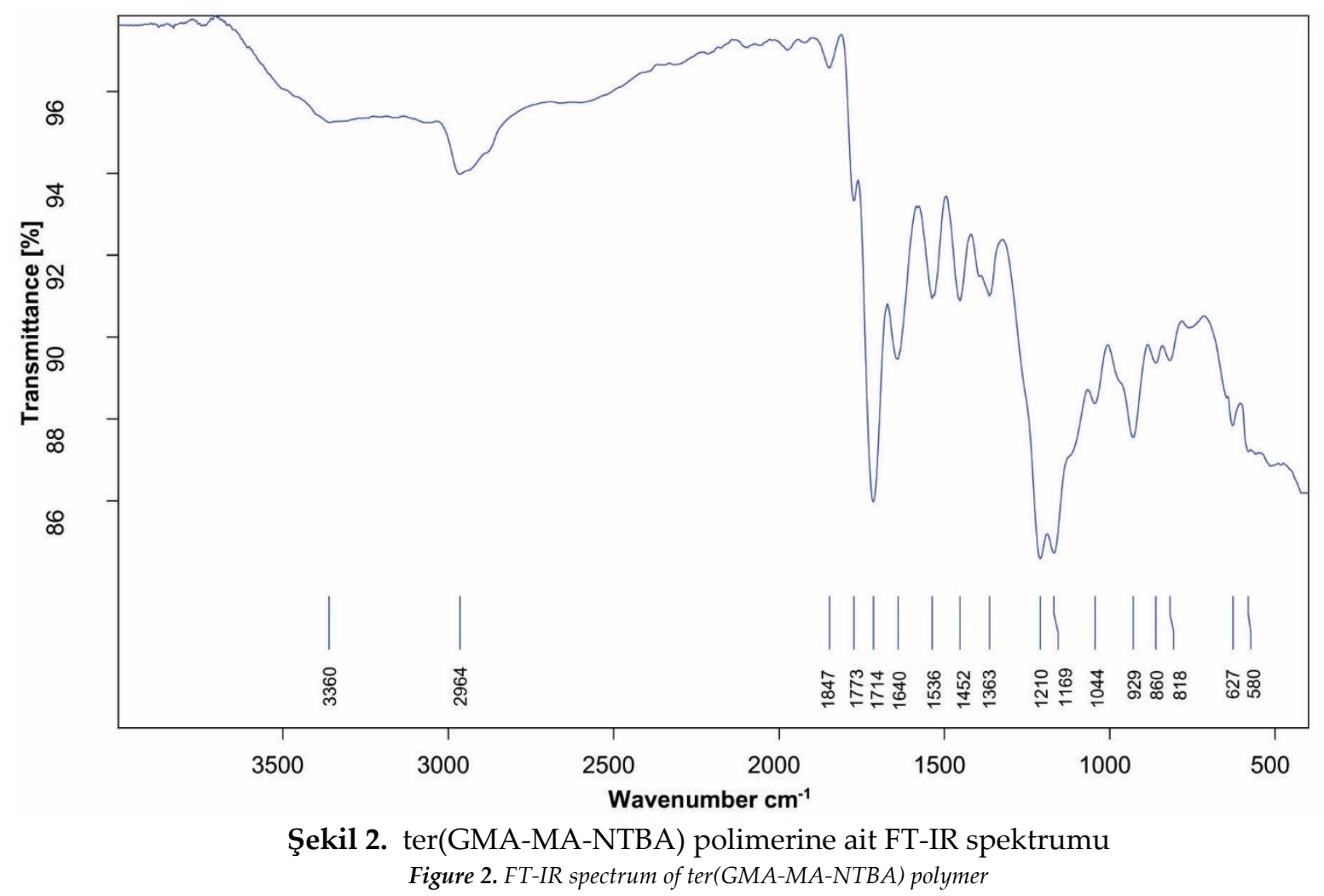

FT-IR spektrumunda maleik anhidrite ait olan $1169 \mathrm{~cm}^{-1}$ de -C-O-C- gerilmesi pikleri görülmektedir (Şengöz, 2014). Maleik anhidritte olan 1847-1773 $\mathrm{cm}^{-1}$ de siklo yapıdaki karbonil grubunun asimetri ve simetrik gerilmeleri, polimerdeki maleik anhidrit grubundan modifikasyon olduğu düşünülmektedir.

$N$-ter-bütil akrilamit'e ait olan pikler; $3360 \mathrm{~cm}^{-1}$ de (sekonder amit, $\left.-\mathrm{NH}\right), 2964 \mathrm{~cm}^{-1}$ de $\left(\mathrm{CH}_{2}\right.$ ve $\mathrm{CH}_{3}$ gruplarının C-H gerilmesi), $1640 \mathrm{~cm}^{-1}$ de (amit karbonili gerilmesi, $-\mathrm{HN}-\mathrm{C}=\mathrm{O}$ ), $1536 \mathrm{~cm}^{-1^{\prime}} \mathrm{de}$ (amit II, -NH) görülmektedir (Şengöz, 2014; Karasakal A., 2015).

\section{ter(GMA-MA-NTBA)'1n '1H-NMR spektrumu ('H-NMR spectrum of ter(GMA-MA-NTBA))}

Sentezlenen ter(GMA-MA-NTBA) polimeri; Nükleer Magnetik Rezonans (1H-NMR) spektroskopisi ile analiz edilmiştir. Şekil 3'teki NTBA-MA-GMA ter-polimerinin $\mathrm{CDCl}_{3}$ çözeltisindeki ${ }^{1} \mathrm{H}$ NMR spektrumundaki; 1,23 ppm'de $N$-ter-butilakrilamid'den gelen ter-butil grubu $\left.\left[-\mathrm{C}_{(\mathrm{CH}}\right)_{3}\right]$ ve glisidil metakrilat'dan gelen metil (- $\left.\mathrm{CH}_{3}\right)$ grubu protonlarına; 2,48 ppm'de $\mathrm{N}$-ter-butilakrilamid'den gelen vinil köküne ait metin grubu $(-\mathrm{CH})$ protonlarına ve glisidil metakrilat'dan gelen epoksi grubuna bağlı metilen $\left(-\mathrm{CH}_{2}\right)$ grubu protonlarına; 3,34-3.55 ppm arasında maleik anhidrit'ten gelen metin grubu $(-\mathrm{CH})$ protonlarına, GMA'nın glisidil kökünden gelen metilen $\left(-\mathrm{CH}_{2}\right)$ grubu protonlarına ve glisidil metakrilat'ın glisidil kökünden gelen metin $(-\mathrm{CH})$ grubu protonlarına; 6,13 ppm'de GMA'daki epoksi grubunun hidrolizinden kaynaklanabilecek hidroksil $(-\mathrm{OH})$ grubu protonlarına; 7,70 ppm'deki pik $N$ ter-butilakrilamid'den gelen amin (-NH) grubu protonlarına aittir (Şengöz, 2014; Karasakal A., 2015). 


ใิ

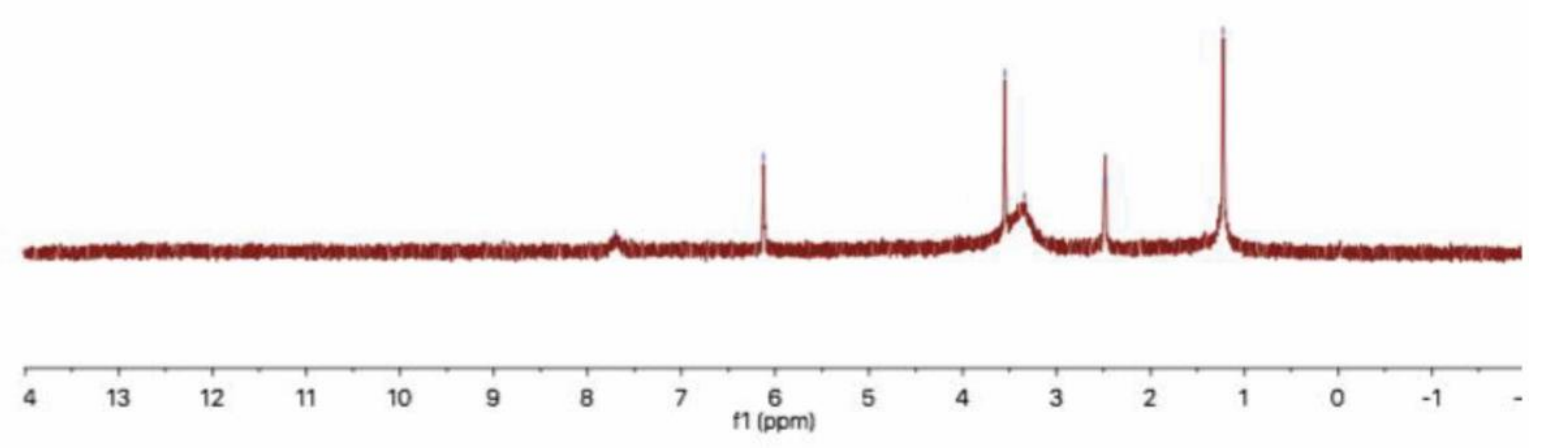

Şekil 3. ter(GMA-MA-NTBA) polimerine ait ${ }^{1} \mathrm{H}-\mathrm{NMR}$ spektrumu Figure 3. ${ }^{1} H-N M R$ spectrum of ter(GMA-MA-NTBA)

\section{ter(GMA-MA-NTBA)'ın SEM görüntüsü (SEM image of ter(GMA-MA-NTBA))}

Sentezlenen ter(GMA-MA-NTBA) polimeri; Taramalı Elektron Mikroskobu (SEM) ile analiz edilmiş ve SEM görüntüsü Şekil 4'te verilmiştir.
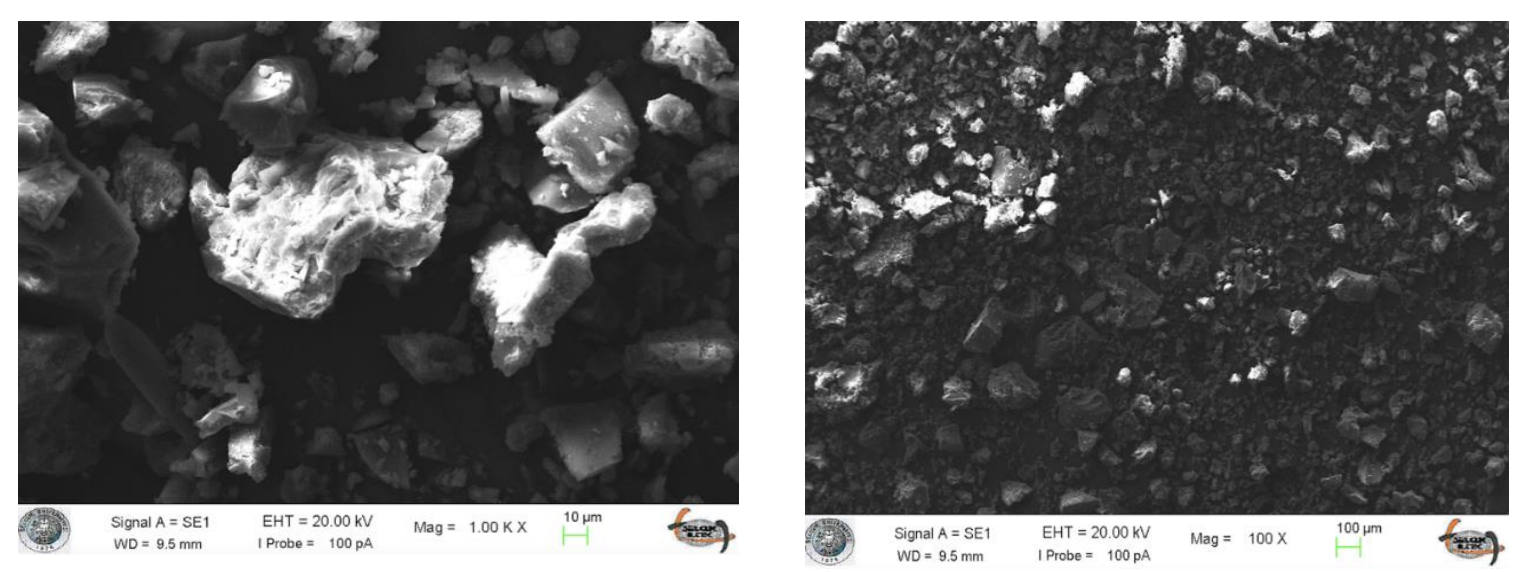

Şekil 4. ter(GMA-MA-NTBA) polimerine ait farklı boyutlardaki SEM görüntüsü Figure 4. SEM images of ter(GMA-MA-NTBA) polymer at different magnifications 
ter(GMA-MA-NTBA)'ın asit sayısı ve viskozimetre (Acid number and viscosimetry of ter(GMA-MANTBA))

ter(GMA-MA-NTBA) polimeri içerisindeki anhidrit grupları birbirleriyle etkileşimiyle karboksil asit oluşturmaktadır. Karboksilik asit; sodyum hidroksit ile titrasyonuyla asit miktarı hesaplanabilmektedir (Şengöz, 2014; Karasakal A., 2015).

Ubbelohde Viskozimetresi kullanılarak ter(GMA-MA-NTBA) polimer çözeltisinin, viskozimetrenin kapiler borusunda işaretlenmiş iki çizgi arasında serbest bırakılarak akış süresi saniye olarak ölçülür. Viskozite suya karşı ölçülürse, bağıl viskozite bulunur. Polimer çözeltileri, yüksek mol kütleleri nedeniyle yüksek viskozite gösterirler. Ölçümlerde moleküller arası etkileşimleri azaltmak ve doğru ölçüm almak için düşük derişimlerde polimer çözeltileri ile çalışılır (Şengöz, 2014; Karasakal A., 2015).

ter(GMA-MA-NTBA) polimeri için asitlik sayısı $587 \mathrm{mg}$ ve limit viskozite [ $\eta$ ] $0.0272 \mathrm{dL} / \mathrm{g}$ olarak hesaplanmıştır. Asitlik sayısı polimer çözeltisinin $\mathrm{NaOH}$ çözeltisiyle titre edildi ve Eşitlik 1 kullanarak belirlendi (Şengöz, 2014).

$\% \mathrm{COOH}=\frac{\left(V_{1}-V_{2}\right) \cdot \mathrm{f} \cdot \mathrm{X} \cdot 100}{m}$

$V_{1}$ : Polimer olan numunedeki harcanan $\mathrm{NaOH}$ hacmi

$V_{2}$ : Polimer olmayan numunedeki harcanan $\mathrm{NaOH}$ hacmi

f: $0.1 \mathrm{~N} \mathrm{NaOH}$ çözeltisinin faktörü

x: 1 mL $0.1 \mathrm{~N}$ olan $\mathrm{NaOH}$ çözeltisine eşdeğer olan karboksil miktarı $(0,0045)$

$m$ : Polimer örneğinin g olarak kütlesi

Bağıl viskozitelerinin belirlenmesi ter(GMA-MA-NTBA) çözeltilerinin ve saf çözücünün aynı Ubbelohde viskozimetresinde aynı koşullarda akış süreleri ölçüldü ve dört tekrar yapıldı. Eşitlik 2 kullanılarak bağıl viskozite $\left(\eta_{\mathrm{r}}\right)$ değeri hesaplandı ve $\eta_{\mathrm{r}}$ 'den spesifik viskozite $\left(\eta_{\mathrm{sp}}\right)($ Şengöz, 2014).

$$
\begin{aligned}
& \eta_{\mathrm{r}}=\mathrm{n} / \mathrm{n}_{\mathrm{o}}=\mathrm{t} / \mathrm{t}_{\mathrm{o}} \\
& \eta_{\mathrm{sp}}=\eta_{\mathrm{r}}-1
\end{aligned}
$$

$\eta_{\text {sp }} / C$ oranının derişime karşı olan (C) değerlerinin grafiği çizilip, Eşitlik 4'ü kullanarak doğrunun dikey ekseni kestiği noktadan limit viskozite sayısı $\left([\eta]=\left[\eta_{\mathrm{sp}} / \mathrm{C}\right]_{c \rightarrow 0}\right.$ ) hesaplandı (Şengöz, 2014).

$$
\eta_{\mathrm{sp}} / \mathrm{C}=[\eta]+\mathrm{k}^{1}\left[\eta^{2}\right] \mathrm{C}
$$

\section{Mikrokapsüllerin Hazırlanması ve Karakterizasyonu (Preparation and Characterization of Microcapsules)}

Kapsülleme ajanı olan kitosan (3 g) 150 mL \%2'lik asetik asit çözeltisine ilave edilerek 1 gün boyunca karıştırılarak çözüldü. ter(GMA-MA-NTBA) polimeri agat havanda ögüüüldükten sonra kitosan çözeltisine çözelti içerisindeki oranı (ağırlıkça) \%0.25 ve \%0.50 olacak şekilde ilave edildi ve karışımlar 3 saat karıştırılarak taneciklerin çözelti içerisinde dağılması sağlandı. Büret içerisine alınan karışım damlalar halinde koagülasyon çözeltisine $\left(25^{\circ} \mathrm{C}\right)$ (1 litre için; $600 \mathrm{~mL}$ metanol, $400 \mathrm{~mL}$ su ve $\left.120 \mathrm{~g} \mathrm{NaOH}\right)$ ilave edildi ve hidrojel halindeki küresel küreler koagülasyon çözeltisi içerisinde manyetik karıştırıcı ile 150 rpm'de karıştırıldı (Sargın ve Arslan, 2015; Sargın ve diğ., 2015; Pal ve diğ., 2013; Abou El-Reash ve

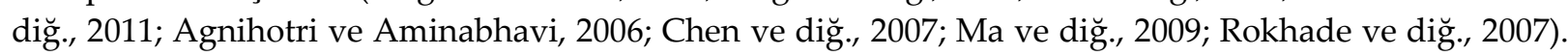
Mikrokapsüller koagülasyon çözeltisinde içinde 1 gün bekletildikten sonra; nüçe hunisinden süzülerek pH'sı nötral oluncaya kadar yıkandı. Yıkanan mikrokapsüller sslak halde çapraz bağlama işlemine alındı. Çapraz bağlama işlemi için kitosanın amin gruplarıyla Schiff bazı oluşturan gluteraldehit kullanıldı. $90 \mathrm{~mL}$ metanol ve $0.9 \mathrm{~mL}$ gluteraldehit çözeltisi içerisinde mikrokapsüller 6 saat boyunca $70^{\circ} \mathrm{C}^{\prime}$ da geri soğutucu altında karıştırıldı (150 rpm'de). 6 saat çapraz bağlanmaya bırakılan mikrokapsüller soğumaya bırakıldı, süzülerek saf suyla yıkandı. Süzülen mikrokapsüller oda sıcaklığında kurumaya bırakıldı (Sargın ve diğ., 2015). Mikrokapsüllerin karakterizasyonunda, FT-IR ve 
SEM analiz yöntemleri kullanılmıştır. FT-IR ile kompozitin fazlarını ve kompozitin fonksiyonel gruplarını görüntülemekte kullanılmıştır. SEM görüntüleri ile mikrokapsül içine dağılan polimerin varlığı ve dağılımı görüntülenmiş ve kapsüllerin boyutu belirlenmiştir.

\section{Kitosan/ter(GMA-MA-NTBA) mikrokapsülün FT-IR spektrumu (FT-IR spectrum of chitosan/ter(GMA-MA-NTBA)}

Hazırlanan Kitosan/ter(GMA-MA-NTBA) mikrokapsülün; Kızılötesi Spektroskopi (FT-IR) ile analiz edilmiş ve FT-IR spektrumu Şekil 5'te verilmiştir.

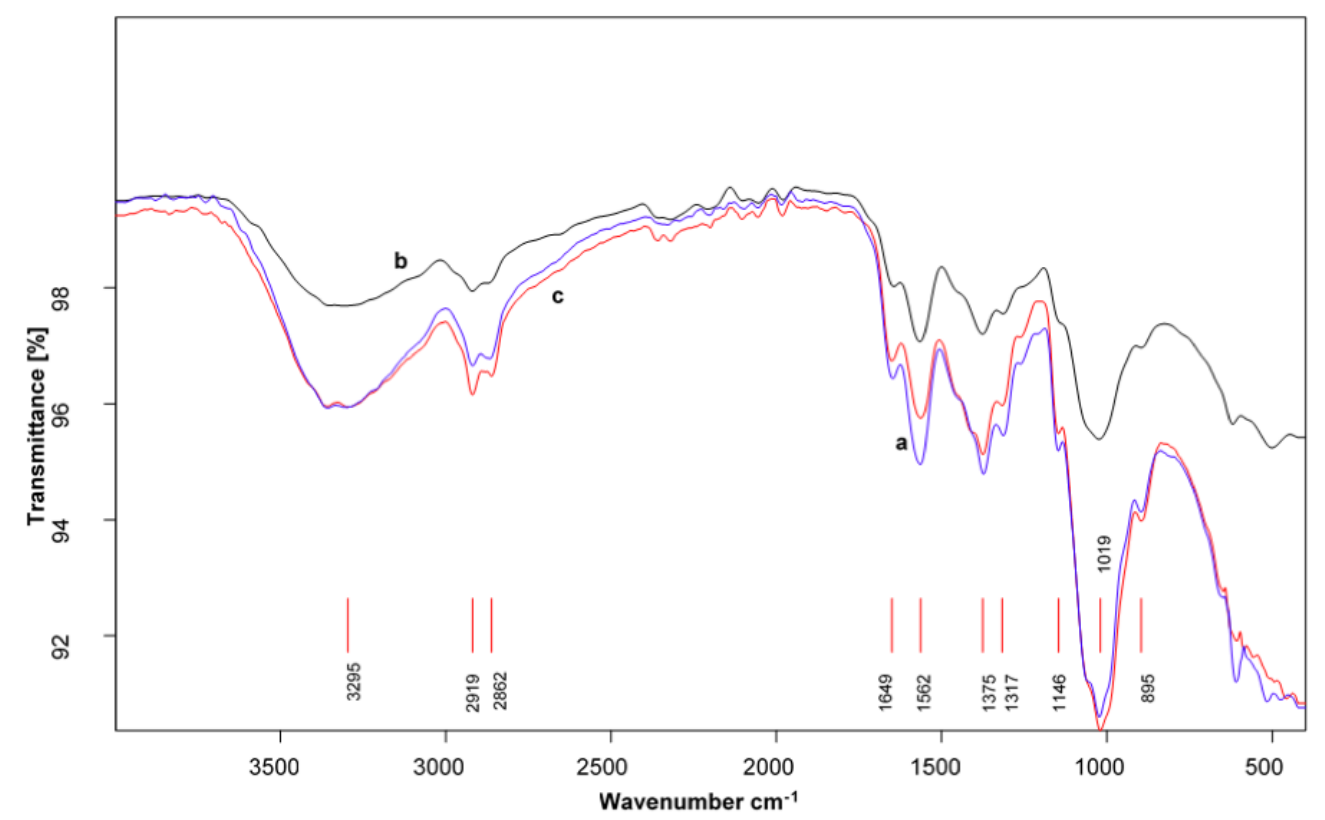

Şekil 5. FT-IR spektrumu: a) kitosan; b) \%0.25 ter(GMA-MA-NTBA) içerikli kitosan; c) \%0.50 ter(GMAMA-NTBA) içerikli kitosan

Figure 5. FT-IR spectra: a) chitosan; b) chitosan with $0.25 \%$ ter(GMA-MA-NTBA) content; c) chitosan with $0.50 \%$ ter(GMA-MA-NTBA) content

FT-IR spektrumda; 2919-2862 $\mathrm{cm}^{-1}$ aralığında $\left(\mathrm{CH}_{3}\right.$ ve $\mathrm{CH}_{2}$ gruplarına ait $\mathrm{C}-\mathrm{H}$ gerilmesi), 1146 $\mathrm{cm}^{-1}$ de maleik anhidrite ait (-C-O-C- gerilmesi) pikleri terpolimerinin mikrokapsül içindeki varlığını doğrulamakta olduğu görülmektedir (Şengöz, 2014; Karasakal A., 2015). Maleik anhidritte olan 1847$1773 \mathrm{~cm}^{-1}$ de siklo yapidaki karbonil grubunun asimetri ve simetrik gerilmeleri, kitosan ile polimer etkileştikten sonra kaybolduğu, polimerdeki maleik anhidrit grubundan modifikasyon olduğu düşünülmektedir.

$3295 \mathrm{~cm}^{-1}$ de ise $N$-ter-Bütil Akrilamit'e ait olan pik görülmektedir (sekonder amit, $-\mathrm{NH}$ ), 2862 $\mathrm{cm}^{-1}$ de $\left(\mathrm{CH}_{2}\right.$ ve $\mathrm{CH}_{3}$ gruplarının $\mathrm{C}-\mathrm{H}$ gerilmesi), $1649 \mathrm{~cm}^{-1}$ de (Amit karbonili gerilmesi, $-\mathrm{HN}-\mathrm{C}=\mathrm{O}$ ), $1562 \mathrm{~cm}^{-1}$ de (Amit II, -N-H), pikleri görülmektedir (Şengöz, 2014). ter(GMA-MA-NTBA) polimerinin mikrokapsül içinde yer aldığı sonucunu doğrulamaktadır. Amit miktarı arttıkça $1649 \mathrm{~cm}^{-1}$ deki pik şiddetinin arttığ1 görülmektedir. 3700-3000 $\mathrm{cm}^{-1}$ arasında kitosanın yapısındaki $-\mathrm{O}-\mathrm{H}$ ve $-\mathrm{N}-\mathrm{H}$ titreşimleri görülmektedir. $3000-2850 \mathrm{~cm}^{-1}$ arasında C-H titreşimleri görülmektedir.

Kitosan/ter(GMA-MA-NTBA) mikrokapsülün SEM görüntüsü (SEM image of Chitosan/ter(GMAMA-NTBA))

Hazırlanan Kitosan / ter(GMA-MA-NTBA) mikrokapsülün; yüzey yapılarına ait SEM görüntüleri farklı miktarlarda ter(GMA-MA-NTBA) içeren sırasıyla Şekil 6'da verilmiştir. Kitosanın yüzeyindeki boşlukların, kitosanın ter-(GMA-MA-NTBA) polimerleri ile mikrokapsül oluşturmasından sonra 
dolduğu ve polimer içeren mikrokapsüllerin daha kompakt bir yüzeye sahip olduğu SEM görüntülerinde de anlaşılmaktadır. Mikrokapsüller için boyut ortalaması boş kapsül, $\% 0.25$ ve $\% 0.50$ ter(GMA-MA-NTBA) polimer içeren kapsüllerin sırasıyla $862 \mu \mathrm{m}, 948 \mu \mathrm{m}$ ve $1094 \mu \mathrm{m}$ olarak belirlenmiştir. 

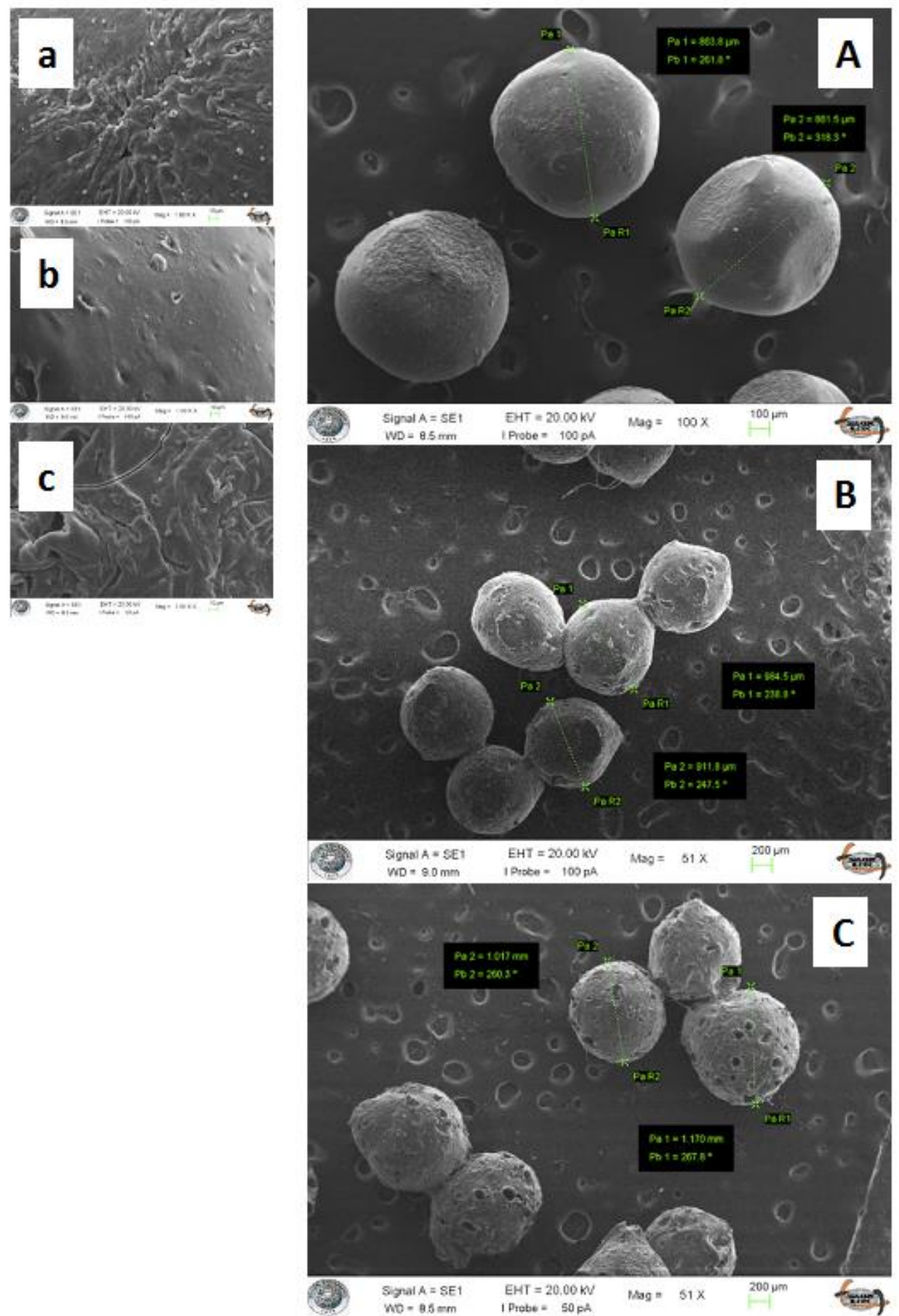

Şekil 6. SEM görüntüsü: kitosan boncuklar (a, A), \%0.25 ter(GMA-MA-NTBA) içerikli kitosan mikrokapsüller (b, B); \%0.50 ter(GMA-MA-NTBA) içerikli kitosan mikrokapsüller (c, C)

Figure 6. SEM images: Chitosan beads $(a, A)$, chitosan microcapsules with $0.25 \%$ ter(GMA-MA-NTBA) content (b, B), chitosan microcapsules with $0.50 \%$ ter(GMA-MA-NTBA) content (c, C)

\section{SONUÇ ve TARTIŞMALAR (RESULTS and DISCUSSIONS)}

İlk kez sentezlenmiş olan ter(GMA-MA-NTBA) polimerle $\% 0.25$ ve $\% 0.50$ içerecek şekilde farklı oranlarda kitosan katkılı mikrokapsüllerin hazırlanması gerçekleştirilmiştir. Hazırlanan yeni nesil kitosan/ter(GMA-MA-NTBA) polimer mikrokapsüllerin boyut ortalaması boş kapsül: $862 \mu \mathrm{m}, \% 0.25$ polimer içeren: $948 \mu \mathrm{m}$ ve \% 0.50 polimer içeren: $1094 \mu \mathrm{m}$ olarak SEM görüntülerinde tespit edilmiştir. Mikrokapsül üretiminde, kitosan-ter(GMA-MA-NTBA) karışımı kitosanın hidrojel oluşturabilme özelliği 
sayesinde mikrokapsül haline getirilmiş ve mekanik ve yapısal kararlılığı sağlayabilmek için kitosanın aldehitlerle Schiff bazı oluşturması esas alınarak kitosan matriks çapraz bağlanmıştır (Tirkistani, 1998; Hassan ve diğ., 2018). Yapısal kararlı kitosan/ter(GMA-MA-NTBA) polimer mikrokapsüller, kitosanın sahip olduğu $-\mathrm{OH},-\mathrm{NH}_{2},-\mathrm{NH}(\mathrm{CO}) \mathrm{CH}_{3}$ gruplarının yanında terpolimerin sahip olduğu butilakrilamid, maleik anhidrit ve glisidil metakrilat'tan gelen fonksiyonel gruplar sayesinde farklı organik moleküllerle, inorganik katyonik veya anyonik türlerle, metal katyonlarıyla, oksianyonlarla etkileşim gösterebilir. Kitosan/ter(GMA-MA-NTBA) mikrokapsüller, farklı yöntem ve yaklaşımlarla sentezlenebilir, ilaç taşıma ve kontrollü ilaç salınım çalışmalarında kullanılabilir. Biyouyumlu ve doğal bir polimer olan kitosanın, farklı sentetik ve biyouyumlu polimerle birlikte mikrokapsül üretiminde kullanılması amacıyla, yeni çalışmalara ihtiyaç vardır.

\section{KATKI BELİRTME (ACKNOWLEDGEMENT)}

ter(GMA-MA-NTBA) polimerin hazırlanmasında katkı, öneri ve yardımları için Selçuk Üniversitesi Fen Fakültesi Kimya Bölümünden sayın Doç. Dr. Ahmet OKUDAN'a çok teşekkür ederim. Bu çalışmaya destek sağlayan Selçuk Üniversitesi Bilimsel Araştırma Projeleri (BAP) (proje no: 17401077) Koordinatörlüğüne teşekkür ederim.

\section{KAYNAKLAR (REFERENCES)}

Abou El-Reash Y. G., Otto M., Kenawy I. M., Ouf A. M., 2011, “Adsorption of $\mathrm{Cr}(\mathrm{VI})$ and $\mathrm{As}(\mathrm{V})$ Ions by Modified Magnetic Chitosan Chelating Resin", International Journal of Biological Macromolecules, Cilt 49, ss. 513-522.

Agnihotri S. A., Aminabhavi T. M., 2006, “Novel Interpenetrating Network Chitosan-Poly(ethylene oxide-g-acrylamide) Hydrogel Microspheres for the Controlled Release of Capecitabine", International Journal of Pharmaceutics, Cilt 324, ss. 103-115.

Akkaya R., Ulusoy U., 2008, “Adsorptive Features of Chitosan Entrapped in Polyacrylamide Hydrogel for $\mathrm{Pb}^{2+}, \mathrm{UO}^{2+}$, and $\mathrm{Th}^{4+\prime}$, Journal of Hazardous Materials, Cilt 151, ss. 380-388.

Anirudhan T. S., Rijith S., 2009, “Glutaraldehyde Cross-linked Epoxyaminated Chitosan as an Adsorbent for the Removal and Recovery of Copper(II) from Aqueous Media", Colloids and Surfaces A: Physicochemical and Engineering Aspects, Cilt 351, ss. 52-59.

Atia A. A., 2005, "Studies on the Interaction of Mercury(II) and Uranyl(II) with Modified Chitosan Resins", Hydrometallurgy, Cilt 80, ss. 13-22.

Babu V. R., Hosamani K. M., Aminabhavi T. M., 2008, "Preparation and in vitro Release of Chlorothiazide Novel $\mathrm{pH}$-sensitive Chitosan- $N, N^{\prime}$-dimethylacrylamide Semi-Interpenetrating Network Microspheres", Carbohydrate Polymers, Cilt 71, ss. 208-217.

Bayramoglu G., Arica M. Y., Bektas S., 2007, "Removal of Cd(II), Hg(II), and Pb(II) Ions from Aqueous Solution Using p(HEMA/Chitosan) Membranes", Journal of Applied Polymer Science, Cilt 106, ss. 169-177.

Chen J., Sun J., Yang L., Zhang Q., Zhu H., Wu H., Hoffman A. S., Kaetsu I., 2007 “Preparation and Characterization of a Novel IPN Hydrogel Membrane of Poly(Nisopropylacrylamide)/carboxymethyl Chitosan (PNIPAAM/CMCS)", Radiation Physics and Chemistry, Cilt 76, ss. 1425-1429.

Chen C. Y., Chang T. H., Kuo J. T., Chen Y. F., Chung Y. C., 2008, "Characteristics of Molybdateimpregnated Chitosan Beads (MICB) in Terms of Arsenic Removal from Water and the Application of a MICB-packed Column to Remove Arsenic from Wastewater", Bioresource Technology, Cilt 99, ss. 7487-7494.

Dinçer S., Köseli V., Kesim H., Rzaev Z. M. O. Pişkin E., 2002, "Radical Copolymerization of NIsopropylacrylamide with Anhydrides of Maleic and Citraconic Acids", European Polymer Journal, Cilt 38, ss. 2143-2152. 
Don T. M., Chen H. R., 2005, “Synthesis and Characterization of AB-crosslinked Graft Copolymers Based on Maleilated Chitosan and N-isopropylacrylamide", Carbohydrate Polymers, Cilt 61, ss. 334-347.

Dotto G. L., Moura J. M., Cadaval T. R. S., Pinto L. A. A., 2013, “Application of Chitosan Films for the Removal of Food Dyes from Aqueous Solutions by Adsorption", Chemical Engineering Journal, Cilt 214, ss. 8-16.

Dragan E. S., Lazar M. M., Dinu M. V., Doroftei F., 2012a, “Macroporous Composite IPN Hydrogels Based on Poly(acrylamide) and Chitosan with Tuned Swelling and Sorption of Cationic Dyes", Chemical Engineering Journal, Cilt 204-206, ss. 198-209.

Dragan E. S., Perju M. M., Dinu M. V., 2012b, "Preparation and Characterization of IPN Composite Hydrogels Based on Polyacrylamide and Chitosan and their Interaction with Ionic Dyes", Carbohydrate Polymers, Cilt 88, ss. 270-281.

Ekberov, O. H., Basan, S., 1995, Polimer Kimyası Laboratuvarı, 1. Baskı, Cumhuriyet Üniversitesi Yayınları, No: 58 , Sivas.

Guibal E., Jansson-Charrier M., Saecudo I., Le Cloirec P., 1995, "Enhancement of Metal Ion Sorption Performances of Chitosan: Effect of the Structure on the Diffusion Properties", Langmuir, Cilt 11, ss. 591-598.

Hassan, M. A., Omer, A. M., Abbas, E., Baset, W. M. A., Tamer, T. M., 2018, "Preparation, Physicochemical Characterization and Antimicrobial Activities of Novel Two Phenolic Chitosan Schiff Base Derivatives", Scientific Reports, Cilt 8, ss. 11416.

Kaplan Can H., Rzaev Z. M. O., 2012, “H-Bonding Effect in Radical Terpolymerization of Maleic Anhydride, Acrylic acid(Methyl acrylate) and Vinyl Acetate", Hacettepe Journal of Biology $\mathcal{E}$ Chemistry, Cilt 40, Sayı 4, ss. 427-443.

Karasakal A., 2015, Aromatik Yapı İçeren Polimerlerin Sentezi ve Karakterizasyonu, Yüksek Lisans Tezi, Selçuk Üniversitesi, Fen Bilimleri Enstitüsü, Konya.

Kestwal D. B, Kestwal R. M., Chiang B. H., Karve M. S., 2011, “Development of Dip-strip Sucrose Sensors: Application of Plant Invertase Immobilized in Chitosan-Guar Gum, Gelatin and Polyacrylamide Films", Sensors and Actuators B, Cilt 160, ss. 1026-1033.

Klumperman B., 2010, "Mechanistic Considerations on Styrene - Maleic Anhydride Copolymerization Reactions", Polymer Chemistry, Cilt 1, ss. 558-562.

Kumar M. N. V. R., Muzzarelli R. A. A., Muzzarelli C., Sashiwa H., Domb A.J., 2004, "Chitosan Chemistry and Pharmaceutical Perspectives", Chemical Reviews, Cilt 104, ss. 6017-6084.

Kumar M., Bijay P. T., Vinod K. S., 2009, “Crosslinked Chitosan/Polyvinyl Alcohol Blend Beads for Removal and Recovery of Cd(II) from Wastewater", Journal of Hazardous Materials, Cilt 172, ss. 1041-1048.

Liu T., Wang Z. L., Zhao L., Yang X., 2012, “Enhanced Chitosan/Fe0-nanoparticles Beads for Hexavalent Chromium Removal from Wastewater", Chemical Engineering Journal, Cilt 189-190, ss. 196-202.

Ma L., Lu W., Wen J., 2009, “Encapsulation of Lactate Dehydrogenase in Carbon Nanotube Doped Alginate-Chitosan Capsules", Journal of Molecular Catalysis B: Enzymatic, Cilt 56, ss. 102-107.

Nie F. Q., Xu, Z. K., Wan, L. S., Ye, P., W, J., 2004, “Acrylonitrile-based Copolymers Containing Reactive Groups: Synthesis and Preparation of Ultrafiltration Membranes", Journal of Membrane Science, Cilt 230, ss. 1-11.

Pal A., Pan S., Saha S., 2013, "Synergistically Improved Adsorption of Anionic Surfactant and Crystal Violet on Chitosan Hydrogel Beads", Chemical Engineering Journal, Cilt 217, ss. 426-434.

Pillai C. K. S., Paul W., Sharma C. P., 2009, "Chitin and Chitosan Polymers: Chemistry, Solubility and Fiber Formation", Progress in Polymer Science, Cilt 34, ss. 641-678.

Piron E., Accominoti M., Domard A., 1997, "Interaction between Chitosan and Uranyl Ions. Role of Physical and Physicochemical Parameters on the Kinetics of Sorption", Langmuir, Cilt 13, ss. 1653-1658. 
Rzaev Z. M. O., 1999, “Complex-radical Terpolymerization of Glycidyl(Methyl) Methacrylates, Styrene, and Maleic Anhydride", Journal of Polymer Science: Part A: Polymer Chemistry, Cilt 37, ss. 10951102.

Rokhade A. P., Patil S. A., Aminabhavi T. M., 2007, "Synthesis and Characterization of SemiInterpenetrating Polymer Network Microspheres of Acrylamide Grafted Dextran and Chitosan for Controlled Release of Acyclovir", Carbohydrate Polymers, Cilt 67, ss. 605-613.

Sargin I., Arslan G., 2015, "Chitosan/Sporopollenin Microcapsules: Preparation, Characterisation and Application in Heavy Metal Removal", International Journal of Biological Macromolecules, Cilt 75, ss. 230-238.

Sargin I., Kaya M., Arslan G., Baran T., Ceter T., 2015, "Preparation and Characterisation of Biodegradable Pollen-Chitosan Microcapsules and its Application in Heavy Metal Removal", Bioresource Technology, Cilt 177, ss. 1-7.

Sharmin N., Khan R. A., Dussault D., Salmieri S., Akter N., Lacroix M., 2012, "Effectiveness of Silane Monomer and Gamma Radiation on Chitosan Films and PCL-Based Composites", Radiation Physics and Chemistry, Cilt 81, ss. 932-935.

Şengöz O., 2014, Maleik Anhidrit İçeren Kopolimerlerin Sentezi, Karakterizasyonu ve Modifikasyonu, Yüksek Lisans Tezi, Selçuk Üniversitesi, Fen Bilimleri Enstitüsü Konya.

Tirkistani, F. A. A., 1998, "Thermal Analysis of Some Chitosan Schiff Bases", Polymer Degradation and Stability, Cilt 60, ss. 67-70.

Tran C. D., Duri S., Ambra Delneri A., Franko M., 2013, “Chitosan-Cellulose Composite Materials: Preparation, Characterization and Application for Removal of Microcystin", Journal of Hazardous Materials, Cilt 252-253, ss. 355-366.

Wang W. B., Huang D. J., Kang Y. R., Wang A. Q., 2013, “One-step in situ Fabrication of a Granular Semi-IPN Hydrogel Based on Chitosan and Gelatin for Fast and Efficient Adsorption of $\mathrm{Cu}^{2+}$ Ion", Colloids and Surfaces B: Biointerfaces, Cilt 106, ss. 51-59.

Wei Z., Wang C., Zou S., Liu H., Tong Z., 2012, “Chitosan Nanoparticles as Particular Emulsifier for Preparation of Novel pH-responsive Pickering Emulsions and PLGA Microcapsules", Polymer, Cilt 53, ss. 1229-1235.

Won S. L., Lee H. C., Jeong Y. G., Min B. G., Lee S. C., 2009, “Preparation and Acid Dye Adsorption Behavior of Polyurethane/Chitosan Composite Foams", Fibers and Polymers, Cilt 10, ss. 636-642.

Wu L. G., Shen J. N., Chen H. L., Gao C. J., 2006. “CO2 Facilitated Transport Through an Acrylamide and Maleic Anhydride Copolymer Membrane", Desalination, Cilt 193, ss. 313-320.

Xu R., Zhou Q., Li F., Zhang B., 2013, “Laccase Immobilization on Chitosan/poly(vinylalcohol) Composite Nanofibrous Membranes for 2,4-dichlorophenol Removal", Chemical Engineering Journal, Cilt 222, ss. 321-329.

Yin L., Fei L., Cui F., Tang C., Yin C., 2007, “Superporous Hydrogels Containing Poly(acrylic acid-coacrylamide)/O-carboxymethyl Chitosan Interpenetrating Polymer Networks", Biomaterials, Cilt 28, ss. $1258-1266$.

Zhao S., Zhou F., Li L., Cao M., Zuo D., Liu H., 2012, “Removal of Anionic Dyes from Aqueous Solutions by Adsorption of Chitosan-based Semi-IPN Hydrogel Composites", Composites: Part B, Cilt 43, ss. $1570-1578$.

Zhou C., Wu Q., 2011, “A Novel Polyacrylamide Nanocomposite Hydrogel Reinforced with Natural Chitosan Nanofibers", Colloids and Surfaces B: Biointerfaces, Cilt 84, ss. 155-162. 